Boyan Zlatanov

\title{
Best proximity points in modular function spaces
}

Received: 19 January 2015 / Accepted: 6 August 2015 / Published online: 26 August 2015

(C) The Author(s) 2015. This article is published with open access at Springerlink.com

\begin{abstract}
We generalize the notion of best proximity points in the context of modular function spaces. We have found sufficient conditions for the existence and uniqueness of best proximity points for cyclic maps in modular function spaces. We present an application of the main result for cyclic integral operators in Orlicz function spaces, endowed with an Orlicz function modular.
\end{abstract}

Mathematics Subject Classification $47 \mathrm{H} 10 \cdot 54 \mathrm{H} 25 \cdot 45 \mathrm{D} 05 \cdot 46 \mathrm{~A} 80$

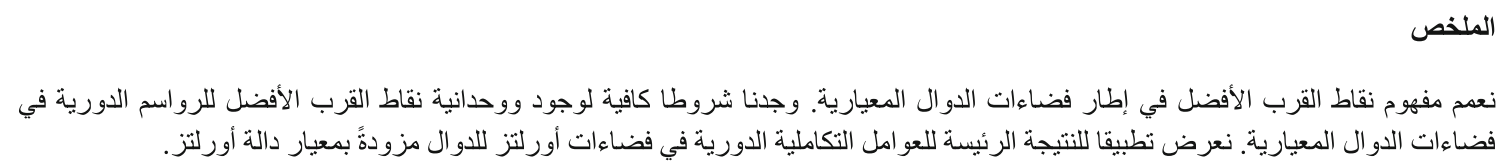

\section{Introduction}

A fundamental result in fixed point theory is the Banach contraction principle in Banach spaces or in complete metric spaces. Fixed point theory is an important tool for solving equations $T x=x$ for mapping $T$ defined on subsets of metric or normed spaces. It is widely applied to nonlinear integral equations and differential equations.

One kind of a generalization of the Banach contraction principle is the notion of cyclic maps [7]. Because a non-self mapping $T: A \rightarrow B$ does not necessarily have a fixed point, one often attempts to find an element $x$ which is in some sense closest to $T x$. Best proximity point theorems are relevant in this perspective. The notion of best proximity point is introduced in [2]. This definition is more general than the notion of cyclic maps [7], in the sense that if the sets intersect then every best proximity point is a fixed point. A sufficient condition for the existence and uniqueness of the best proximity points in uniformly convex Banach spaces is given in [2].

Besides the idea of defining a norm and considering a Banach space, another direction of generalization of the Banach contraction principle is based on considering an abstractly given functional defined on a linear space, which controls the growth of the members of the space. This functional is usually called modular and defines a modular space. The theory of modular spaces was initiated by Nakano [15] in connection with the theory of ordered spaces, which was further generalized by Musielak and Orlicz [14]. Modular function spaces are a subclass of the modular spaces. The study of the geometry of modular function spaces was initiated by

B. Zlatanov $(\bowtie)$

Faculty of Mathematics and Informatics, Plovdiv University "Paisii Hilendarski”, 24 “Tzar Assen” str., 4000 Plovdiv, Bulgaria E-mail: bzlatanov@gmail.com 
Kozlowski [8-10]. Fixed point results in modular function spaces were obtained first by Khamsi et al. [5]. Further development of the theory of fixed points in modular function spaces can be found in the exhaustive references of the survey article [11] and in the book [4]. Kozlowski has contributed a lot towards the study of modular function spaces, both on his own and with his collaborators.

We have tried to generalize the idea of best proximity points in modular function spaces and to present an application for integral operators in Orlicz function spaces, endowed with an Orlicz function modular.

\section{Modular function spaces}

We will give a brief summary of basic definitions and results about modular function spaces. We will follow the survey paper [11], where the needed definitions and results for fixed point theory in modular function spaces are presented in the most concise manner. For an extensive study of fixed point theory in modular function spaces and the geometry of modular function spaces, we refer to [4] and for modular space to $[14,15]$. When we do not indicate a reference item in this section, it means that we are following [11].

Let $\Omega$ be a nonempty set and $\Sigma$ be a nontrivial $\sigma$-algebra of subsets of $\Omega$. Let $\mathcal{P}$ be a $\delta$-ring of subsets of $\Omega$, such that $E \cap A \in \mathcal{P}$ for any $E \in \mathcal{P}$ and $A \in \Sigma$. Let us assume that there exists an increasing sequence of sets $K_{n} \in \mathcal{P}$, such that $\Omega=\cup K_{n}$. By $\mathcal{E}$, we denote the linear space of all simple functions with supports from $\mathcal{P}$. By $\mathcal{M}_{\infty}$, we will denote the space of all extended measurable functions, i.e., all functions $f: \Omega \rightarrow[-\infty, \infty]$ such that there exists a sequence $\left\{g_{n}\right\} \subset \mathcal{E},\left|g_{n}\right| \leq|f|$ and $g_{n}(\omega) \rightarrow f(\omega)$ for all $\omega \in \Omega$. By $\mathbf{1}_{A}$, we denote the characteristic function of the set $A$.

Definition 2.1 Let $\rho: \mathcal{M}_{\infty} \rightarrow[0, \infty]$ be a nontrivial convex and even function. We say that $\rho$ is a regular convex function pseudomodular if:

(i) $\rho(0)=0$;

(ii) $\rho$ is monotone, i.e., $|f(\omega)| \leq|g(\omega)|$ for all $\omega \in \Omega$ implies $\rho(f) \leq \rho(g)$, where $f, g \in \mathcal{M}_{\infty}$;

(iii) $\rho$ is orthogonaly subadditive, i.e., $\rho\left(f \mathbf{1}_{A \cup B}\right) \leq \rho\left(f \mathbf{1}_{A}\right)+\rho\left(f \mathbf{1}_{B}\right)$, where $A, B \in \Sigma$ such that $A \cap B \neq \emptyset$, $f \in \mathcal{M}_{\infty}$;

(iv) $\rho$ has the Fatou property, i.e., $\left|f_{n}(\omega)\right| \uparrow|f(\omega)|$ for all $\omega \in \Omega$ implies $\rho\left(f_{n}\right) \uparrow \rho(f)$, where $f \in \mathcal{M}_{\infty}$;

(v) $\rho$ is order continuous in $\mathcal{E}$, i.e., $g_{n} \in \mathcal{E}$ and $\left|g_{n}(\omega)\right| \downarrow 0$ implies $\rho\left(g_{n}\right) \downarrow 0$.

Similarly to that in the case of measure spaces, we say that a set $A \in \Sigma$ is $\rho$-null if $\rho\left(g \mathbf{1}_{A}\right)=0$ for every $g \in \mathcal{E}$. We say that a property holds $\rho$-almost everywhere if the exceptional set is $\rho$-null. As usual, we identify any pair of measurable sets whose symmetric difference is $\rho$-null as well as any pair of measurable functions differing only on a $\rho$-null set. With this in mind, we define

$$
\mathcal{M}(\Omega, \sigma, \mathcal{P}, \rho)=\left\{f \in \mathcal{M}_{\infty} ;|f(\omega)|<\infty \rho-\text { a.e. }\right\},
$$

where each $f \in \mathcal{M}(\Omega, \sigma, \mathcal{P}, \rho)$ is actually an equivalence class of functions equal to $\rho$ a.e. rather than an individual function. Where no confusion exists, we will write $\mathcal{M}$, instead of $\mathcal{M}(\Omega, \sigma, \mathcal{P}, \rho)$.

Definition 2.2 Let $\rho$ be a regular convex function pseudomodular.

(1) We say that $\rho(0)$ is a regular convex function semimodular if $\rho(\alpha f)=0$ for every $\alpha>0$ implies $f=0$ $\rho$-a.e.

(2) We say that $\rho$ is a regular convex function modular if $\rho(f)=0$ implies $f=0 \rho$-a.e.

The class of all nonzero regular convex function modular defined on $\Omega$ will be denoted by $\Re$.

Let us denote $\rho(f, E)=\rho\left(f 1_{E}\right)$ for $f \in \mathcal{M}, E \in \Sigma$. It is easy to prove that $\rho(f, E)$ is a function pseudomodular in the sense of Definition 2.1.1 in [10] (more precisely, it is a function pseudomodular with the Fatou property). Therefore, we can use all results of the standard theory of modular function spaces as per the framework defined by Kozlowski [8-10]; see also Musielak and Orlicz [14] for the basics of the general modular theory.

Definition 2.3 Let $\rho$ be a convex function modular.

(a) A modular function space is the vector space $L_{\rho}(\Omega, \Sigma)$, or briefly $L_{\rho}$, defined by $L_{\rho}=\{f \in \mathcal{M}: \rho$ $(\lambda f) \rightarrow 0$ as $\lambda \rightarrow 0\}$.

(b) The following formula defines a norm in $L_{\rho}$ (frequently called Luxemburg norm): $\|f\|_{\rho}=$ $\inf \left\{\alpha>0: \rho\left(\frac{f}{\alpha}\right) \leq 1\right\}$. 
For the rest of the article, if we state something about a norm we will mean Luxemburg norm $\|\cdot\|_{\rho}$, which is generated by the modular $\rho$.

In this way, Lebesgue, Orlicz, Musielak-Orlicz, Lorentz and Orlicz-Lorentz are examples of modular function spaces.

In the following theorem, we recall some of the basic properties of modular function spaces.

Theorem 2.4 Let $\rho \in \mathfrak{R}$.

(1) $\left(L_{\rho},\|f\|_{\rho}\right)$ is complete and the norm $\|\cdot\|_{\rho}$ is a monotone w.r.t the natural order in $\mathcal{M}$.

(2) $\left\|f_{n}\right\|_{\rho} \rightarrow 0$ iff $\rho\left(\alpha f_{n}\right) \rightarrow 0$ for every $\alpha>0$.

(3) If $\rho\left(\alpha f_{n}\right) \rightarrow 0$ for an $\alpha$, then there exists a subsequence $\left\{g_{n}\right\}$ of $\left\{f_{n}\right\}$ such that $g_{n} \rightarrow 0 \rho$-a.e.

(4) If $\left\{f_{n}\right\}$ converges uniformly to $f$ on a set $E \in \mathcal{P}$, then $\rho\left(\alpha\left(f_{n}-f\right), E\right) \rightarrow 0$ for every $\alpha>0$.

(5) Let $f_{n} \rightarrow f \rho$-a.e. There exists a nondecreasing sequence of sets $H_{k} \in \mathcal{P}$ such that $H_{k} \uparrow \Omega$ and $f_{n}$ converge uniformly to $f$ on every $H_{k}$ (Egoroff Theorem).

(6) $\rho(f) \leq \lim \inf \rho\left(f_{n}\right)$ whenever $f_{n} \rightarrow f \rho$-a.e. (note that this property is equivalent to the Fatou property).

(7) Defining $L_{\rho}^{0}\left\{f \in L_{\rho}: \rho(f, \cdot)\right.$ is order continuous $\}$ and $E_{\rho}=\left\{f \in L_{\rho}: \lambda f \in L_{\rho}^{0}\right.$ for every $\left.\lambda>0\right\}$, we have

(a) $L_{\rho} \supset L_{\rho}^{0} \supset E_{\rho}$;

(b) $E_{\rho}$ has the Lebesgue property, i.e., $\rho\left(\alpha f, D_{k}\right) \rightarrow 0$ for $\alpha>0, f \in E_{\rho}$ and $D_{k} \downarrow \emptyset$;

(c) $E_{\rho}$ is the closure of $\mathcal{E}$ (in the sense of $\|\cdot\|_{\rho}$ ).

The next definition gives generalizations of the classical notions for normed spaces in the context of modular function spaces.

Definition 2.5 Let $L_{\rho} \in \mathfrak{R}$.

(a) We say that $\left\{f_{n}\right\}$ is a $\rho$-convergent to $f$ and we write $f_{n} \rightarrow f(\rho)$ if and only if $\rho\left(f_{n}-f\right) \rightarrow 0$.

(b) A sequence $\left\{f_{n}\right\}_{n=1}^{\infty} \subset L_{\rho}$ is called $\rho$-Cauchy if for any $\varepsilon>0$ there exists $N \in \mathbb{N}$, such that for any $m>n \geq N$ there holds the inequality $\rho\left(f_{m}-f_{n}\right)<\varepsilon$.

(c) The modular function space $L_{\rho}$ is called $\rho$-complete if any $\rho$-Cauchy sequence is $\rho$-convergent.

(d) A set $B \subset L_{\rho}$ is called $\rho$-closed if for any sequence of $f_{n} \in B$, the convergence $f_{n} \rightarrow f(\rho)$ implies that $f$ belongs to $B$.

(e) A set $B \subset L_{\rho}$ is called $\rho$-bounded if its $\rho$-diameter $\delta_{\rho}(B)=\sup \{\rho(f-g): f, g \in B\}<\infty$.

(f) A set $B \subset L_{\rho}$ is called $\rho$-a.e. closed if for any $\left\{f_{n}\right\}$ in $C$ in which $\rho$-a.e. converges to some $f$, then we must have $f \in C$.

(g) Let $A, B \subset L_{\rho}$. We define the $\rho$-distance between the sets $A$ and $B$ by

$$
d_{\rho}(A, B)=\inf \{\rho(f-g): f \in A, g \in B\} .
$$

If $A$ consists of a single element $f$, then $d_{\rho}(f, B)=\inf \{\rho(f-g): g \in B\}$.

(h) We say that $\rho$ has the $\Delta_{2}$-property if $\sup _{n \in \mathbb{N}} \rho\left(2 f_{n}, D_{k}\right) \rightarrow 0$, whenever $D_{k} \downarrow \emptyset$ and $\sup _{n \in \mathbb{N}} \rho\left(f_{n}, D_{k}\right) \rightarrow$ 0 .

(i) [4, p.116] A function modular $\rho \in \mathfrak{R}$ is called uniformly continuous if for any $L>0$ and $\varepsilon>0$ there exists $\delta=\delta(L, \varepsilon)>0$, such that if $\rho(x) \leq L$ and $\rho(y)<\delta$ there holds the inequality $|\rho(x+y)-\rho(x)|<\varepsilon$.

Theorem 2.6 Let $\rho \in \mathfrak{R}$, then $L_{\rho}$ is $\rho$-complete.

Theorem 2.7 Let $\rho \in \Re$. The following conditions are equivalent

(a) $\rho$ has $\Delta_{2}$.

(b) $L_{\rho}^{0}$ is a linear subspace of $L_{\rho}$.

(c) $L_{\rho}=L_{\rho}^{0}=E_{\rho}$.

(d) If $\rho\left(f_{n}\right) \rightarrow 0$, then $\rho\left(2 f_{n}\right) \rightarrow 0$.

(e) If $\rho\left(\alpha f_{n}\right) \rightarrow 0$ for an $\alpha>0$, then $\left\|f_{n}\right\|_{\rho} \rightarrow 0$, i.e., the modular convergence is equivalent to the norm convergence.

Let us mention that the $\rho$-convergence does not imply $\rho$-Cauchy, since $\rho$ does not satisfy the triangle inequality. If $\rho$ has $\Delta_{2}$-property, then $\rho$-convergence implies $\rho$-Cauchy.

The generalization of convexity properties for Banach spaces is investigated for modular function spaces in [6]. As demonstrated in [11], one concept of uniform convexity for Banach spaces generates several different types of uniform convexity in modular function spaces. This is due primarily to the fact that in general, the modular function is not homogeneous. 
Definition 2.8 Let $\rho \in \mathfrak{R}$ and $i \in\{1,2\}$. Let $r>0, \varepsilon>0$. Define

$$
D_{i}(r, \varepsilon)=\left\{(f, g): f, g \in L_{\rho}, \rho(f) \leq r, \rho(g) \leq r, \rho\left(\frac{f-g}{i}\right) \geq \varepsilon r\right\} .
$$

Let $\delta_{i}(r, \varepsilon)=\inf \left\{1-\frac{1}{r} \rho\left(\frac{f+g}{2}\right):(f, g) \in D_{i}(r, \varepsilon)\right\}>0$ if $D_{i}(r, \varepsilon) \neq \emptyset$ and $\delta_{i}(r, \varepsilon)=1$ if $D_{i}(r, \varepsilon)=\emptyset$.

(i) We say that $\rho$ satisfies (UCi) if for any $r>0, \varepsilon>0$ there holds the inequality $\delta_{i}(r, s)>0$.

(ii) We say that $\rho$ satisfies (UUCi) if for every $s \geq 0, \varepsilon>0$ there exists $\eta_{i}(s, \varepsilon)>0$, depending on $s$ and $\varepsilon$ such that

$$
\delta_{i}(r, s)>\eta_{i}(s, \varepsilon)>0 \text { for } r>s .
$$

If $\rho$ is (UC1), we obtain that the inequality

$$
\rho\left(\frac{x+y}{2}\right) \leq r\left(1-\delta_{1}(r, \varepsilon)\right)
$$

holds for every $\rho(x), \rho(y) \leq r$ and $\rho(x-y) \geq r \varepsilon$.

Proposition 2.9 The following conditions characterize the relationship between the notions that are defined in Definition 2.8

(1) (UUCi) implies (UCi) for $i \in 1,2$.

(2) $\delta_{1}(r, \varepsilon) \leq \delta_{2}(r, \varepsilon)$.

(3) (UC1) implies (UC2).

(4) (UUC1) implies (UUC2).

(5) If $\rho \in \Re$, then (UUC1) and (UUC2) are equivalent.

(6) If $\rho$ is homogeneous (e.g. is a norm), then all conditions (UC1), (UC2), (UUC1) and (UUC2) are equivalent.

We recall that $M$ is called an Orlicz function, provided $M$ is even, convex and continuous nondecreasing in $[0, \infty)$ function with $M(0)=0, M(t)>0$ for any $t \neq 0$. Let $M$ be an Orlicz function and let $(\Omega, \Sigma, \mu)$ be a measure space. Let us consider the space $L^{0}(\Omega)$ consisting of all measurable real-valued functions on $\Omega$ and define for every $f \in L^{0}(\Omega)$ the Orlicz function modular $M(f)=\int_{\Omega} M(f(t)) \mathrm{d} \mu(t)$.

Definition 2.10 The Orlicz space $L_{M}(\Omega, \Sigma, \mu)$ is the space of all classes of equivalent $\mu$-measurable functions $f: \Omega \rightarrow \mathbb{R}$ over the measure space $(\Omega, \Sigma, \mu)$, such that $\widetilde{M}(\lambda f) \rightarrow 0$ as $\lambda \rightarrow 0$ or equivalently $\widetilde{M}\left(\frac{f}{\lambda}\right)<\infty$ for some $\lambda>0$.

The function $\widetilde{M}$ is a regular convex function modular and it is called Orlicz function modular. An extensive study of Orlicz spaces can be found in $[1,12,13,16,17]$.

If $M(t)=|t|^{p}, p \geq 1$, we obtain the space $L_{p}(\Omega, \Sigma, \mu)$. The most common examples of Orlicz spaces are the sequence spaces $\ell_{M}$, the function spaces $L_{M}(0,1)$ and $L_{M}(0, \infty)$ that correspond to the cases: $\Omega$ countable union of atoms of equal mass, $\Omega=[0,1]$ and $\Omega=(0, \infty)$, and $\mu$ the usual Lebesgue measure.

We say that $M$ satisfies the $\Delta_{2}$-condition if there exist constants $C, t_{0}>0$, such that $M(2 t) \leq C M(t)$ for any $t \geq t_{0}$. It is easy to observe that if $M$ satisfies the $\Delta_{2}$-condition, then the Orlicz function modular $\widetilde{M}$ has the $\Delta_{2}$ property.

If we restrict to the Orlicz space $L_{M}(0,1)$, then the Orlicz function modular is defined by $\widetilde{M}(f)=$ $\int_{0}^{1} M(f(s)) \mathrm{d} \mu(s)$. We will denote the corresponding modular function space by $L_{\widetilde{M}}(0,1)$. When $M=|t|^{p}$, we will denote $L_{\widetilde{M}}(0,1)$ by $L_{\widetilde{p}}(0,1)$.

Definition 2.11 [7] Let $A, B$ be two sets. The map $T: A \cup B \rightarrow A \cup B$ is called a cyclic map if $T(A) \subseteq B$ and $T(B) \subseteq A$.

We will generalize the notion of best proximity point in a metric spaces [2] for modular function spaces.

Definition 2.12 Let $\rho \in \mathfrak{R}, A_{1}, A_{2} \subset L_{\rho}$ be two subsets and $T: A_{1} \cup A_{2} \rightarrow A_{1} \cup A_{2}$ be a cyclic map. A point $\xi \in A_{i}$ is called a $\rho$-best proximity point of the cyclic map $T$ in $A_{i}$ if $\rho(\xi, T \xi)=d_{\rho}\left(A_{1}, A_{2}\right)$.

We will prove a general result about $\rho$-best proximity points for modular function spaces. An application of this general result for Orlicz function spaces, endowed with an Orlicz function modular, will be presented. 


\section{Main result}

Definition 3.1 Let $\rho \in \mathfrak{R}, A, B \subseteq L_{\rho}$ be subsets. The map $T: A \cup B \rightarrow A \cup B$ is called a cyclic $\rho$-contraction if it is a cyclic map and there exists $k \in(0,1)$, such that the inequality

$$
\rho(T x-T y) \leq k \rho(x-y)+(1-k) d_{\rho}(A, B)
$$

holds for every $x \in A, y \in B$.

Theorem 3.2 Let $\rho \in \mathfrak{R}$. Assume that $\rho$ satisfies (UC1), has the $\Delta_{2}$-property and is uniformly continuous. Let $A, B \subseteq L_{\rho}$ be $\rho$-closed, convex subsets, $A \cup B$ be $\rho$-bounded and $T: A \cup B \rightarrow A \cup B$ be a cyclic $\rho$-contraction. Then there exists a unique $x \in A$ such that $x$ is a $\rho$-best proximity point of $T$ in $A, T^{2} x=x$ and for any $x_{0} \in A$ the point $x$ is a $\rho$-limit of the sequence $\left\{T^{2 n} x_{0}\right\}_{n=1}^{\infty}$.

\section{Auxiliary results}

For the rest of the article, $k$ will be the constant from (2), and for simplicity of the notations we denote $d_{\rho}(A, B)$ by $d_{\rho}$.

Lemma 4.1 Let $\rho \in \mathfrak{R}, A, B \subseteq L_{\rho}$ be subsets, $A \cup B$ be $\rho$-bounded and $T: A \cup B \rightarrow A \cup B$ be a cyclic $\rho$-contraction. Then there holds

$$
\lim _{n \rightarrow \infty} \rho\left(T^{n+1} x-T^{n} x\right)=d_{\rho}
$$

for every $x \in A \cup B$.

Proof Let $x \in A \cup B$ be arbitrary. Let us denote $A_{n}(x)=\rho\left(T^{n+1} x-T^{n} x\right)-d_{\rho}$. The inequality (2) can be written in an equivalent form $\rho(T x-T y)-d_{\rho} \leq k\left(\rho(x-y)+d_{\rho}\right)$. After applying $n$-times (2), we obtain the chain of inequalities

$$
\begin{aligned}
A_{n}(x) & =\rho\left(T^{n+1} x-T^{n} x\right)-d_{\rho} \leq k\left(\rho\left(T^{n} x-T^{n-1} x\right)-d_{\rho}\right) \\
& \leq k^{2}\left(\rho\left(T^{n-1} x-T^{n-2} x\right)-d_{\rho}\right) \leq \cdots \leq k^{n}\left(\rho(T x-x)-d_{\rho}\right) .
\end{aligned}
$$

Thus from $k \in(0,1)$, it follows that $\lim _{n \rightarrow \infty} \rho\left(T^{n+1} x-T^{n} x\right)=d_{\rho}$.

Lemma 4.2 Let $\rho \in \Re, A, B \subseteq L_{\rho}$ be $\rho$-closed convex subsets, $A \cup B$ be $\rho$-bounded and $T: A \cup B \rightarrow A \cup B$ be a cyclic $\rho$-contraction. Fix $x \in A \cup B$. Then for every $\varepsilon>0$, there exists $N_{0} \in \mathbb{N}$, such that for every $m, n \geq N_{0}, n+m$ is an odd number and there holds the inequality $\rho\left(T^{n} x-T^{m} x\right)<d_{\rho}+\varepsilon$.

Proof Without loss of generality, we may assume that $n<m$. By assumption, $m-n$ is an odd number. Let us take $M=\sup \left\{\rho\left(x-T^{2 j-1} x\right): j \in \mathbb{N}\right\}$. It is easy to observe that $M>d_{\rho}$. From the $\rho$-boundedness of the set $A \cup B$, it follows that $M<+\infty$. Let $\varepsilon>0$ be arbitrary. There exists $N_{0} \in \mathbb{N}$, such that the inequality $k^{n}\left(M-d_{\rho}\right)<\varepsilon$ holds true for every $n \geq N_{0}$. From (4) for every $m>n \geq N_{0}$, we get the inequalities

$$
\begin{aligned}
\rho\left(T^{n} x-T^{m} x\right) & \leq k^{n} \rho\left(x-T^{m-n} x\right)+\left(1-k^{n}\right) d_{\rho} \\
& =d_{\rho}+k^{n}\left(\rho\left(x-T^{m-n} x\right)-d_{\rho}\right) \\
& \leq d_{\rho}+k^{n}\left(M-d_{\rho}\right)<d_{\rho}+\varepsilon .
\end{aligned}
$$

Lemma 4.3 Let $\rho \in \Re$. Assume that $\rho$ satisfies $(U C 1)$, and has the $\Delta_{2}$-property, and let $A \subset L_{\rho}$ be a $\rho$-closed and convex subset, $B \subset L_{\rho}$ be $\rho$-closed subset and $A \cup B$ be $\rho$-bounded. Let the sequences $\left\{x_{n}\right\}_{n=1}^{\infty},\left\{z_{n}\right\}_{n=1}^{\infty} \subset$ $A$ and $\left\{y_{n}\right\}_{n=1}^{\infty} \subset B$ be such that:

(i) $\lim _{n \rightarrow \infty} \rho\left(z_{n}-y_{n}\right)=d_{\rho}$;

(ii) for every $\varepsilon>0$ there exists $N_{0} \in \mathbb{N}$ such that for every $m>n \geq N_{0}$ there holds the inequality $\rho\left(x_{m}-y_{n}\right) \leq d_{\rho}+\varepsilon$.

Then for every $\varepsilon>0$, there exists $N_{1} \in \mathbb{N}$, such that for every $m>n \geq N_{1}$, there holds the inequality $\rho\left(x_{m}-z_{n}\right)<\varepsilon$. 
Proof Take $\gamma=\sup \{\rho(x, y): x \in A, y \in B\}$. Assume the contrary, i.e., there exists $\varepsilon_{0}>0$, such that for every $k \in \mathbb{N}$ there are $m_{k}>n_{k} \geq k$, so that $\rho\left(x_{m_{k}}-z_{n_{k}}\right) \geq \varepsilon_{0}$. For any two $0<r_{1}<r_{2}$ and $\varepsilon_{0}>0$, there holds the inclusion $D_{1}\left(r_{1}, \varepsilon_{0} / r_{1}\right) \subseteq D_{1}\left(r_{2}, \varepsilon_{0} / r_{2}\right)$. Consequently, we have the inequality $\delta\left(r_{1}, \varepsilon_{0} / r_{1}\right) \geq$ $\delta\left(r_{2}, \varepsilon_{0} / r_{2}\right)$. Let us put $\eta=\inf \left\{\delta_{1}\left(d_{\rho}+\varepsilon, \frac{\varepsilon_{0}}{d_{\rho}+\varepsilon}\right), \varepsilon \in(0, \gamma]\right\}$, then $\eta \geq \delta_{1}\left(d_{\rho}+\gamma, \frac{\varepsilon_{0}}{d_{\rho}+\gamma}\right)$. From the assumption that $\rho$ is (UC1), it follows that $\eta>0$. Choose $\tau_{0} \in(0, \gamma)$, such that $\frac{\tau_{0}}{d_{\rho}+\tau_{0}}<\eta$. The function $f(t)=\frac{t}{d_{\rho}+t}:[0,+\infty) \rightarrow[0,+\infty)$ is an increasing function and therefore for any $\varepsilon \in\left(0, \tau_{0}\right)$ there hold the inequalities

$$
\frac{\varepsilon}{d_{\rho}+\varepsilon}<\frac{\tau_{0}}{d_{\rho}+\tau_{0}}<\eta \leq \delta_{1}\left(d_{\rho}+\varepsilon, \frac{\varepsilon_{0}}{d_{\rho}+\varepsilon}\right) .
$$

From (5), we get that the inequality

$$
\left(d_{\rho}+\varepsilon\right)\left(1-\delta_{1}\left(d_{\rho}+\varepsilon, \frac{\varepsilon_{0}}{d_{\rho}+\varepsilon}\right)\right)<d_{\rho}
$$

holds for every $\varepsilon \in\left(0, \tau_{0}\right)$.

Let $\varepsilon \in\left(0, \tau_{0}\right)$ be arbitrarily chosen. For this $\varepsilon$, there exists $N_{0} \in \mathbb{N}$ such that for any $m_{k}>n_{k} \geq N_{0}$ there holds the inequality $\rho\left(x_{m_{k}}-y_{n_{k}}\right) \leq d_{\rho}+\varepsilon$. For this $\varepsilon$, there exists $N_{2} \in \mathbb{N}$ such that for any $n_{k} \geq N_{2}$ there holds the inequality $\rho\left(z_{n_{k}}-y_{n_{k}}\right) \leq d_{\rho}+\varepsilon$. Take $N_{3}=\max \left\{N_{0}, N_{2}\right\}$. Put $f=y_{n_{k}}-z_{n_{k}}$ and $g=y_{n_{k}}-x_{m_{k}}$ in (1), for $m_{k}>n_{k} \geq N_{3}$. Then we have $\rho(f) \leq d_{\rho}+\varepsilon, \rho(g) \leq d_{\rho}+\varepsilon$ and

$$
\rho(f-g)=\rho\left(x_{m_{k}}-z_{n_{k}}\right) \geq \varepsilon_{0}=\left(d_{\rho}+\varepsilon\right) \frac{\varepsilon_{0}}{\left(d_{\rho}+\varepsilon\right)} .
$$

Using the convexity of the set $A,(1)$ and (6), we get the inequalities

$$
\begin{aligned}
\rho\left(y_{n_{k}}-\frac{x_{m_{k}}+z_{n_{k}}}{2}\right) & =\rho\left(\frac{y_{n_{k}}-z_{n_{k}}+y_{n_{k}}-x_{m_{k}}}{2}\right)=\rho\left(\frac{f+g}{2}\right) \\
& \leq\left(d_{\rho}+\varepsilon\right)\left(1-\delta_{1}\left(d_{\rho}+\varepsilon, \frac{\varepsilon_{0}}{d_{\rho}+\varepsilon}\right)\right)<d_{\rho},
\end{aligned}
$$

which is a contradiction. Therefore for every $\varepsilon>0$, there exists $N_{1} \in \mathbb{N}$, such that for every $m>n \geq N_{1}$ there holds the inequality $\rho\left(x_{m}-z_{n}\right)<\varepsilon$.

In a similar fashion, we can prove the following lemma.

Lemma 4.4 Let $\rho \in \mathfrak{R}$. Assume that $\rho$ satisfies $(U C 1)$, and has the $\Delta_{2}$-property, and let $A$ be a $\rho$-closed and convex subset of $L_{\rho}, B$ be $\rho$-closed subset of $L_{\rho}$ and $A \cup B$ be $\rho$-bounded. If the sequences $\left\{x_{n}\right\}_{n=1}^{\infty},\left\{z_{n}\right\}_{n=1}^{\infty} \subset A$ and $\left\{y_{n}\right\}_{n=1}^{\infty} \subset B$ be such that:

(i) $\lim _{n \rightarrow \infty} \rho\left(z_{n}-y_{n}\right)=d_{\rho}$,

(ii) $\lim _{n \rightarrow \infty} \rho\left(x_{n}-y_{n}\right)=d_{\rho}$,

then $\lim _{n \rightarrow \infty} \rho\left(x_{n}-z_{n}\right)=0$.

Lemma 4.5 Let $\rho \in \Re$. Let $\rho$ has the $\Delta_{2}$-property, be uniformly continuous, $A, B \subset L_{\rho}$ be subsets and $A \cup B$ be $\rho$-bounded. If the sequences $\left\{x_{n}\right\}_{n=1}^{\infty},\left\{z_{n}\right\}_{n=1}^{\infty} \subset A$ and $\left\{y_{n}\right\}_{n=1}^{\infty} \subset B$ be such that:

(i) $\lim _{n \rightarrow \infty} \rho\left(z_{n}-x_{n}\right)=0$,

(ii) $\lim _{n \rightarrow \infty} \rho\left(z_{n}-y_{n}\right)=d_{\rho}$,

then $\lim _{n \rightarrow \infty} \rho\left(x_{n}-y_{n}\right)=d_{\rho}$.

We would like to mention that if $\rho$ satisfies the triangle inequality, the proof is trivial and we do not need the assumption that $\rho$ is uniform continuous. 
Proof Let $\varepsilon>0$ be arbitrarily chosen. There exists $N_{0} \in \mathbb{N}$, such that for every $n \geq N_{0}$ there holds the inequality $\rho\left(y_{n}-z_{n}\right)<d_{\rho}+\frac{\varepsilon}{3}$. From the uniform continuity of $\rho$, it follows that there exists $\delta>0$, such that for any for any $u \in L_{\rho}$, which satisfies $\rho\left(u-\left(y_{n}-z_{n}\right)\right)<\delta$, there holds the inequality $\left|\rho(u)-\rho\left(y_{n}-z_{n}\right)\right|<\varepsilon / 6$. From the inequalities $\rho\left(y_{n}-z_{n}\right)<d_{\rho}+\frac{\varepsilon}{3}$ and $\rho\left(\left(\lambda_{0}-1\right)\left(y_{n}-z_{n}\right)\right) \leq\left(\lambda_{0}-1\right) \rho\left(y_{n}-z_{m}\right)$, it follows that we can choose $\lambda_{0} \in(1,2]$ so that the inequality

$$
\rho\left(\lambda\left(y_{n}-z_{n}\right)-\left(y_{n}-z_{n}\right)\right)=\rho\left((\lambda-1)\left(y_{n}-z_{n}\right)\right)<\delta
$$

holds for every $n \geq N_{0}$ and every $\lambda \in\left(1, \lambda_{0}\right]$. Therefore by the uniform continuity of $\rho$, it follows that the inequality

$$
\left|\rho\left(\lambda\left(y_{n}-z_{n}\right)\right)-\rho\left(y_{n}-z_{n}\right)\right|<\frac{\varepsilon}{6}
$$

holds for every $n \geq N_{0}$ and every $\lambda \in\left(1, \lambda_{0}\right]$.

Let us put $\lambda_{1}=1+\alpha_{1}$. There exists $\alpha_{1}>0$ such that $\lambda_{1} \in\left(1, \lambda_{0}\right]$ and there hold the inequalities $\left|\rho\left(\lambda_{1}\left(y_{n}-z_{n}\right)\right)-\rho\left(y_{n}-z_{n}\right)\right|<\frac{\varepsilon}{6}, \frac{\alpha_{1}}{1+\alpha_{1}} \rho\left(y_{n}-z_{n}\right)<\frac{\varepsilon}{6}$. Consequently, we get that the inequality

$$
\begin{aligned}
J_{1} & =\left|\frac{\rho\left(\lambda_{1}\left(y_{n}-z_{n}\right)\right)}{\lambda_{1}}-\rho\left(y_{n}-z_{n}\right)\right| \\
& =\frac{1}{1+\alpha_{1}}\left|\rho\left(\lambda_{1}\left(y_{n}-z_{n}\right)\right)-\rho\left(y_{n}-z_{n}\right)-\alpha_{1} \rho\left(y_{n}-z_{n}\right)\right| \\
& \leq \frac{1}{1+\alpha_{1}}\left|\rho\left(\lambda_{1}\left(y_{n}-z_{n}\right)\right)-\rho\left(y_{n}-z_{n}\right)\right|+\frac{\alpha_{1}}{1+\alpha_{1}}\left|\rho\left(y_{n}-z_{n}\right)\right|<\frac{\varepsilon}{3}
\end{aligned}
$$

holds true for any $n \geq N_{0}$.

From the assumptions $\lim _{n \rightarrow \infty} \rho\left(x_{n}-z_{n}\right)=0$ and that $\rho$ has the $\Delta_{2}$-property, it follows that for $\lambda_{1}>1$ there exists $N_{1}=N_{1}\left(\lambda_{1}\right) \in \mathbb{N}$, such that for any $n \geq N_{1}$ there holds the inequality

$$
\frac{\lambda_{1}-1}{\lambda_{1}} \rho\left(\frac{\lambda_{1}\left(x_{n}-z_{n}\right)}{\lambda_{1}-1}\right)<\frac{\varepsilon}{3} .
$$

Thus for any $n \geq N_{2}=\max \left\{N_{1}, N_{0}\right\}$ using the convexity of the function modular $\rho$, (7) and (8), we obtain the inequality

$$
\begin{aligned}
\rho\left(y_{n}-x_{n}\right) & =\rho\left(y_{n}-z_{n}+z_{n}-x_{n}\right) \\
& =\rho\left(\frac{\lambda_{1}\left(y_{n}-z_{n}\right)}{\lambda_{1}}+\frac{\lambda_{1}-1}{\lambda_{1}} \frac{\lambda_{1}\left(z_{n}-x_{n}\right)}{\lambda_{1}-1}\right) \\
& \leq \frac{\rho\left(\lambda_{1}\left(y_{n}-z_{n}\right)\right)}{\lambda_{1}}+\frac{\lambda_{1}-1}{\lambda_{1}} \rho\left(\frac{\lambda_{1}\left(z_{n}-x_{n}\right)}{\lambda_{1}-1}\right) \\
& \leq d_{\rho}+\frac{2 \varepsilon}{3}+\frac{\varepsilon}{3}=d_{\rho}+\varepsilon .
\end{aligned}
$$

Lemma 4.6 Let $\rho \in \mathfrak{R}$. Assume that $\rho$ satisfies (UC1), and has the $\Delta_{2}$-property, and let $A, B \subseteq L_{\rho}$ be $\rho$-closed, convex subsets, $A \cup B$ be $\rho$-bounded and $T: A \cup B \rightarrow A \cup B$ be a cyclic $\rho$-contraction. Then there hold

$$
\lim _{n \rightarrow \infty} \rho\left(T^{2 n} x-T^{2 n+2} x\right)=0
$$

and

$$
\lim _{n \rightarrow \infty} \rho\left(T^{2 n+1} x-T^{2 n+3} x\right)=0
$$

for every $x \in A \cup B$.

Proof From Lemma 4.1, we have the equalities $\lim _{n \rightarrow \infty} \rho\left(T^{2 n} x-T^{2 n+1} x\right)=d_{\rho}$ and $\lim _{n \rightarrow \infty} \rho\left(T^{2 n+2} x-\right.$ $\left.T^{2 n+1} x\right)=d_{\rho}$. From Lemma 4.4, we get that $\lim _{n \rightarrow \infty} \rho\left(T^{2 n} x-T^{2 n+2} x\right)=0$.

The proof of the second equality is similar. 
Lemma 4.7 Let $\rho \in \mathfrak{R}$. Let $\rho$ has the $\Delta_{2}$-property, be uniformly continuous, $A, B \subseteq L_{\rho}$ be $\rho$-closed and convex subsets, $A \cup B$ be $\rho$-bounded and $T: A \cup B \rightarrow A \cup B$ be a cyclic $\rho$-contraction. Define the sequence $u_{n+1}=T u_{n}=T^{n+1} u$, where $u \in A \cup B$ is arbitrary. If the sequence $\left\{u_{2 n}\right\}_{n=1}^{\infty}$ is $\rho$-convergent to $x$, then $\rho(x-T x)=d_{\rho}$.

Proof Let us denote $z_{n}=u_{2 n}, y_{n}=u_{2 n-1}$ and $x_{n}=x$, for $n \in \mathbb{N}$. The sequences $\left\{z_{n}\right\}_{n=1}^{\infty},\left\{x_{n}\right\}_{n=1}^{\infty}$ and $\left\{y_{n}\right\}_{n=1}^{\infty}$ satisfy the conditions of Lemma 4.5, i.e., $\lim _{n \rightarrow \infty} \rho\left(z_{n}-x_{n}\right)=0$ and $\lim _{n \rightarrow \infty} \rho\left(z_{n}-y_{n}\right)=d_{\rho}$. Therefore,

$$
\lim _{n \rightarrow \infty} \rho\left(x-u_{2 n-1}\right)=\lim _{n \rightarrow \infty} \rho\left(x_{n}-y_{n}\right)=d_{\rho} .
$$

From the inequalities,

$$
d_{\rho} \leq \rho\left(T x-T^{2 n} u\right) \leq k \rho\left(x-T^{2 n-1} u\right)+(1-k) d_{\rho} \leq \rho\left(x-u_{2 n-1}\right)
$$

and (11) we obtain that $\lim _{n \rightarrow \infty} \rho\left(T x-u_{2 n}\right)=\lim _{n \rightarrow \infty} \rho\left(T x-T^{2 n} u\right)=d_{\rho}$. From uniform continuity of $\rho$, we get $\rho(T x-x)=\lim _{n \rightarrow \infty} \rho\left(T x-u_{2 n}\right)=d_{\rho}$.

Lemma 4.8 Let $\rho \in \Re$. Assume that $\rho$ satisfies (UC1), and has the $\Delta_{2}$-property, and let $A, B \subseteq L_{\rho}$ be $\rho$-closed, convex subsets, $A \cup B$ be $\rho$-bounded and $T: A \cup B \rightarrow A \cup B$ be a cyclic $\rho$-contraction. Then for every $x \in A \cup B$, the sequences $\left\{T^{2 n} x\right\}_{n=1}^{\infty}$ and $\left\{T^{2 n+1}\right\}_{n=1}^{\infty}$ are $\rho$-Cauchy sequences.

Proof From Lemma 4.1, we have that $\lim _{n \rightarrow \infty} \rho\left(T^{2 n} x-T^{2 n+1} x\right)=d_{\rho}$. By Lemma 4.2, we have that for every $\varepsilon>0$ there exists $N_{0} \in \mathbb{N}$, such that there holds the inequality

$$
\rho\left(T^{2 m} x-T^{2 n+1} x\right)<d_{\rho}+\varepsilon
$$

for every $m>n \geq N_{0}$. From Lemma 4.3, there exists $N_{1} \in \mathbb{N}$, such that the inequality $\rho\left(T^{2 m} x-T^{2 n} x\right)<\varepsilon$ holds for every $m>n \geq N_{1}$. Therefore, the sequence $\left\{T^{2 n} x\right\}_{n=1}^{\infty}$ is a $\rho$-Cauchy sequence.

The proof that the sequence $\left\{T^{2 n+1}\right\}_{n=1}^{\infty}$ is a $\rho$-Cauchy sequence is similar.

Let us mention that all of Lemmas hold true for $d_{\rho}=0$.

\section{Proof of main result}

Proof Let $d_{\rho}>0$. Let $x_{0} \in A$ be arbitrary. From Lemma 4.8, we have that the sequence $\left\{T^{2 n} x_{0}\right\}_{n=1}^{\infty}$ is a $\rho$-Cauchy sequence. From Theorem 2.6, the space $L_{\rho}$ is $\rho$-complete and therefore the sequence $\left\{T^{2 n} x_{0}\right\}_{n=1}^{\infty}$ is $\rho$-convergent. Let us denote by $x \in A$ the $\rho$ limit of the sequence $\left\{T^{2 n} x_{0}\right\}_{n=1}^{\infty}$. From Lemma 4.7, we get that $x$ is a $\rho$-best proximity point of $T$ in $A$.

From the inequality $\rho\left(T^{2} x-T x\right) \leq k \rho(T x-x)+(1-k) d_{\rho}=d_{\rho}$, we get that $\rho\left(T^{2} x-T x\right)=d_{\rho}$. From Lemma 4.4, it follows that $\rho\left(x-T^{2} x\right)=0$ and therefore $x=T^{2} x$.

Suppose that there exists another $\rho$-best proximity point $y \neq x$. Then, $T^{2} y=y$. From the system of inequalities

$$
\left|\begin{array}{l}
\rho(T x-y) \leq k \rho(x-T y)+(1-k) d_{\rho} \\
\rho(x-T y) \leq k \rho(T x-y)+(1-k) d_{\rho}
\end{array}\right|
$$

we obtain the inequality $(1-k) \frac{\rho(T x-y)+\rho(x-T y)}{2} \leq(1-k) d_{\rho}$. Let us assume that $\rho(T x-y)>d_{\rho}$, then $\rho(x-T y)<d_{\rho}$ which is a contradiction. Therefore, $\rho(T x-y)=\rho(x-T y)=d_{\rho}$. From the equalities $\rho(T x-y)=\rho(T x-x)=d_{\rho}$ and Lemma 4.4, it follows that $x=y$.

If $d_{\rho}=0$, we get that $\left\{T^{2 n} x\right\}_{n=1}^{\infty}$ is a $\rho$-Cauchy sequence. From the $\rho$-completeness of $L_{\rho}$, it follows that there exists $x=\lim _{n \rightarrow \infty} T^{2 n} x \in A$. From Lemma 4.7, we get that $\rho(x-T x)=d_{\rho}=0$, i.e., $x$ is a fixed point for the map $T$ and $x \in A \cap B$.

Suppose that there exists another fixed point $y \neq x$. From the inequality

$$
\rho(x-y)=\rho(T x-T y) \leq k \rho(x-y),
$$

we get a contradiction. Consequently, there exists a unique fixed point $x \in A \cap B$. 
We would like to state a corollary which covers a wide class of modular function spaces.

Corollary 5.1 Let $\rho \in \mathfrak{R}$. Assume that $\rho$ satisfies (UC1), and is uniformly continuous and there exists $C>0$ such that $\rho(2 f) \leq C \rho(f)$ for any $f \in L_{\rho}$. Let $A, B \subseteq L \rho$ be $\rho$-closed, convex subsets, $A \cup B$ be $\rho$-bounded and $T: A \cup B \rightarrow A \cup B$ be a cyclic $\rho$-contraction. Then there there exists a unique $x \in A$ such that $x$ is a $\rho$-best proximity point of $T$ in $A, T^{2} x=x$ and for any $x_{0} \in A$ the point $x$ is a $\rho$-limit of the sequence $\left\{T^{2 n} x_{0}\right\}_{n=1}^{\infty}$.

\section{Best proximity points for integral equations in Orlicz function spaces}

We will apply Theorem 3.2 to study the existence and uniqueness of the best proximity points to a class of integral operators in Orlicz modular space $L_{\widetilde{M}}(0,1)$. For simplicity of the notation, we will denote $L_{\widetilde{M}}(0,1)$ by $L_{\widetilde{M}}$.

Definition 6.1 [4, p.81] A function $\varphi$ is said to be very convex if for any $\varepsilon>0$ and any $u_{0}$ there exists $\delta>0$ such that $\varphi\left(\frac{u-v}{2}\right) \geq \frac{\varepsilon}{2}(\varphi(u)+\varphi(v)) \geq \varepsilon \varphi\left(u_{0}\right)$ implies $\varphi\left(\frac{u+v}{2}\right) \leq \frac{1-\delta}{2}(\varphi(u)+\varphi(v))$.

Definition 6.2 [3] A function $\varphi$ is said to be uniformly convex on the whole $\mathbb{R}$ if for any $a \in(0,1)$ there exists $\delta(a) \in(0,1)$, such that

$$
\varphi\left(\frac{u+a u}{2}\right) \leq(1-\delta(a)) \frac{\varphi(u)+\varphi(a u)}{2} .
$$

If $\varphi$ is uniformly convex, then $\varphi$ is very convex [6]. Examples of uniformly convex Orlicz functions are $M(t)=|t|^{p}, p>1$. Indeed, let us take

$$
\delta(a)=1-\frac{(1+a)^{p}}{\left(1+a^{p}\right)} .
$$

From $a \in(0,1)$ and $p>1$, it follows that $\delta(a) \in(0,1)$. The inequality (13) is equivalent to $|u|^{p}(1+a)^{p} \leq$ $(1-\delta(a))|u|^{p}\left(1+a^{p}\right)$, i.e., $|t|^{p}$ is uniformly convex for every $p>1$.

The uniform convexity of the Orlicz function implies that (UC1) [3]. It is known [6,11] that for Orlicz spaces over a finite, atomless measure space, the Orlicz modular $\widetilde{M}$ is (UC2) if and only if $M$ is very convex. From Proposition 2.9, (UC2) is equivalent to (UC1), provided that the Orlicz function $M$ satisfies the $\Delta_{2}$ condition. Thus, we will replace in the following examples the assumption that $\rho$ is (UC1), to be very convex and to satisfy the $\Delta_{2}$-condition.

It can be proved $[4$, p.116] that in Orlicz spaces over a finite atomless measure, the uniform continuity of the Orlicz function modular is equivalent to the $\Delta_{2}$-condition.

Let us point out a well-known fact that the set $A=\{u: \alpha(x) \leq u(x) \leq \beta(x)\}$, where $u, \alpha, \beta: \mathbb{R} \rightarrow \mathbb{R}$ is convex. Indeed, let $u_{1}, u_{2} \in A$. Then,

$$
\alpha=\mu \alpha+(1-\mu) \alpha \leq \mu u_{1}+(1-\mu) u_{2} \leq \mu \beta+(1-\mu) \beta=\beta .
$$

Example 6.3 Let $M$ be an Oricz function, which is very convex and satisfies the $\Delta_{2}$-condition. Let us consider the modular function space $L_{\widetilde{M}}$. We assume that $\alpha \in L_{\widetilde{M}}$ and let $n \in \mathbb{N}$ be a fixed number. Denote the sets $A=\left\{u \in L_{\widetilde{M}}: \alpha(x) \leq u(x) \leq n \alpha(x)\right\}, B=\left\{v \in L_{\widetilde{M}}:-n \alpha(x) \leq v(x) \leq-\alpha(x)\right\}$. The map $T: A \cup B \rightarrow A \cup B$, defined by

$$
T u(x)= \begin{cases}-\frac{\alpha(x)+u(x)}{2}, & u \in A \\ -\frac{-\alpha(x)+v(x)}{2}, & v \in B\end{cases}
$$

is a cyclic $\widetilde{M}$-contraction and $\alpha(x) \in A$ is a $\widetilde{M}$-best proximity point of $T$ in $A, T(\alpha)=-\alpha, T^{2}(\alpha)=$ $T(-\alpha)=\alpha$.

From the definition of the map $T$, we have that $\alpha(x) \leq \frac{\alpha(x)+u(x)}{2} \leq n \alpha(x)$ and consequently $T(A) \subseteq B$ and $T(B) \subseteq A$.

The sets $A$ and $B$ are convex. 
Let $u, v \in A \cup B$. Then using the fact that $M$ satisfies the $\Delta_{2}$-condition, we get the inequality

$$
\tilde{M}(u-v) \leq \tilde{M}(2 n|\alpha|)<\infty .
$$

Therefore, $\sup \{\tilde{M}(u-v): u, v \in A \cup B\} \leq \widetilde{M}(2 n|\alpha|)<\infty$, i.e., the set $A \cup B$ is $\widetilde{M}$-bounded. From the condition $\alpha>0$, it follows that $d_{\widetilde{M}}(A, B)=\widetilde{M}(2 \alpha)>0$.

Using the convexity of the function $M$, we get the inequality

$$
\begin{aligned}
\widetilde{M}(T u-T v) & =\widetilde{M}\left(\frac{\alpha-v}{2}+\frac{\alpha+u}{2}\right)=\widetilde{M}\left(\frac{2 \alpha}{2}+\frac{u-v}{2}\right) \\
& \leq \frac{\widetilde{M}(2 \alpha)}{2}+\frac{\widetilde{M}(u-v)}{2}=\frac{1}{2} \widetilde{M}(u-v)+\frac{1}{2} d_{\widetilde{M}}
\end{aligned}
$$

and consequently $T$ is a cyclic $\widetilde{M}$-contraction. Thus from Theorem 3.2, it follows that there exists a unique $\widetilde{M}$-best proximity point $u \in A$, such that for any $u_{0} \in A$, the sequence $\left\{T^{2 n} u_{0}\right\}_{n=1}^{\infty}$ is $\widetilde{M}$-convergent to $u \in A$. It is easy to observe that $\alpha \in A$ is a $\widetilde{M}$-best proximity point of $T$ in $A, T(\alpha)=-\alpha, T^{2}(\alpha)=T(-\alpha)=\alpha$.

For simplicity of the notations, let us denote for a function $u:[0,1] \rightarrow \mathbb{R}$

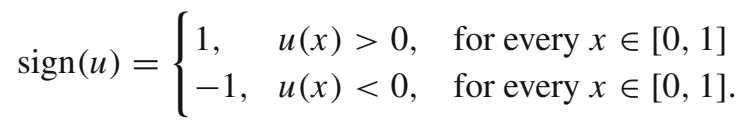

Theorem 6.4 Let $M$ be an Oricz function, which satisfies the $\Delta_{2}$ condition and is very convex. Let $L_{\widetilde{M}}$ be the modular function space generated by $M$. Let $\alpha, \beta, \varphi, \psi \in L_{\widetilde{M}}$. Let us denote $A=\left\{u \in L_{\widetilde{M}}: \alpha(x) \leq u(x) \leq\right.$ $\varphi(x)\}$ and $B=\left\{u \in L_{\tilde{M}}: \psi(x) \leq u(x) \leq \beta(x)\right\}$. Let $K:[0,1] \times[0,1] \rightarrow \mathbb{R}$ and $f, g:[0,1] \rightarrow \mathbb{R}$. Let the map $T$ be defined by

$$
T u(x)=-\operatorname{sign}(u(x))\left(g(x)+\int_{0}^{1} K(x, s) f(u(s)) \mathrm{d} s\right) .
$$

Let the following conditions take place:

(c1) $\beta(x)<0<\alpha(x)$ for every $x \in[0,1]$;

(c2) $\widetilde{M}(\alpha-\beta)>0$

(c3) there exist $k \in(0,1)$ such that the inequalities hold

$$
\tilde{M}(T u-T v) \leq k \widetilde{M}(u-v)+(1-k) d_{\widetilde{M}}
$$

and for any $u \in A, v \in B$

(c4) for any $u \in A$ and $v \in B$ there hold the inclusions $T u \in B$ and $T v \in A$.

Then $T$ is a cyclic $\widetilde{M}$-contraction and there exists a unique $u \in A$, such that $u$ is a $\widetilde{M}$-best proximity point of $T$ in $A, T^{2} u=u$ and for any $u_{0} \in A$ the sequence $\left\{T^{2 n} u_{0}\right\}_{n=1}^{\infty}$ is $\widetilde{M}$-convergent to $u$.

Proof The condition (c4) implies that $T$ is a cyclic map.

Let $u_{1}, u_{2} \in A$ and $v_{1}, v_{2} \in B$. Then using the fact that $M$ satisfies the $\Delta_{2}$-condition, we get the inequalities

$$
\begin{gathered}
\widetilde{M}\left(u_{1}-v_{1}\right) \leq \tilde{M}(|\varphi|+|\psi|) \leq \frac{\widetilde{M}(2|\varphi|)}{2}+\frac{\widetilde{M}(2|\psi|)}{2}<\infty, \\
\widetilde{M}\left(u_{1}-u_{2}\right) \leq \widetilde{M}(|\varphi|+|\varphi|)=\tilde{M}(2|\varphi|)<\infty
\end{gathered}
$$

and

$$
\widetilde{M}\left(v_{1}-v_{2}\right) \leq \tilde{M}(|\psi|+|\psi|)=\tilde{M}(2|\psi|)<\infty .
$$

Therefore, $\sup \{\tilde{M}(u-v): u, v \in A \cup B\}<\infty$, i.e., the set $A \cup B$ is $\widetilde{M}$-bounded.

From conditions (c1) and (c2), it follows that $d_{\widetilde{M}} \geq 0$.

From the inequalities (14), we get that $T$ is a cyclic $\widetilde{M}$-contraction. Thus, all of the conditions of Theorem 3.2 are fulfilled. 
Example 6.5 Let $L_{\widetilde{2}}[0,1]$ be the modular function space, which is generated by the Orlicz function $M(t)=|t|^{2}$. Let us consider the functions $f(x)=|x|, K(x, s)=\frac{x s}{2}$ and $g(x)=\frac{5}{6} x$. Denote the sets $A=\{u \in$ $\left.L_{\widetilde{2}}[0,1]: x \leq u(x) \leq n x\right\}, B=\left\{v \in L_{\widetilde{2}}[0,1]:-n x \leq v(x) \leq-x\right\}$, where $n \in \mathbb{N}$. The map $T: A \cup B \rightarrow$ $A \cup B$, defined by

$$
T u(x)=-\operatorname{sign}(u(x))\left(g(x)+\int_{0}^{1} K(x, s) f(u(s)) \mathrm{d} s\right)
$$

is a cyclic $\widetilde{M}$-contraction and $x \in A$ is a $\widetilde{M}$-best proximity point of $T$ in $A, T(x)=-x, T^{2}(x)=T(-x)=x$.

We will show that the map $T$ satisfies the conditions of Theorem 6.4. The function $M(t)=|t|^{2}$ is an Orlicz function, from the class $|t|^{p}, p \in(1,+\infty)$ and therefore $A \cup B$ is $\widetilde{M}$-bounded and $|t|^{2}$ is uniformly convex. The sets $A$ and $B$ are convex.

For any $t \in\left[t_{0},+\infty\right), t_{0}>0, p>1$ there holds the inequality

$$
(1+t)^{p} \leq\left(1+t_{0}\right)^{p-1}+\left(\frac{1+t_{0}}{t_{0}}\right)^{p-1} t^{p}
$$

Indeed, let us define the function $F(t)=\left(\frac{1+t_{0}}{t_{0}}\right)^{p-1} t^{p}+\left(1+t_{0}\right)^{p-1}-(1+t)^{p}$. From the fact that the function $\frac{1+t}{t}$ is a decreasing function on the interval $(0,+\infty)$, it follows that $F^{\prime}(t) \geq 0$ for every $t \in\left[t_{0},+\infty\right)$. From the equality $F\left(t_{0}\right)=0$, we get that the inequality (15) holds true.

We will check that the map $T$ satisfies the conditions (c1)-(c4).

(c1) For every $x \in[0,1]$ the inequalities $\beta(x)=-x<0<x=\alpha(x)$ hold.

(c2) It is easy to see that

$$
d_{\widetilde{M}}(A, B)=\widetilde{M}(x-(-x))=\int_{0}^{1}|2 x|^{2} \mathrm{~d} x>0
$$

As $p=2$, we get that $q=2$, which is the solution of the equation $\frac{1}{p}+\frac{1}{q}=1$.

(c3) We will need the constants: $a=\frac{\widetilde{M}\left(\frac{5}{6} x-\left(-\frac{5}{6} x\right)\right)}{2}=\frac{\int_{0}^{1}\left|\frac{5}{3} x\right|^{2} \mathrm{~d} x}{2}=\frac{25}{27}, b=d_{\widetilde{M}}(A, B)=\frac{4}{3}$ and $c=$ $2 \int_{0}^{1}\left(\int_{0}^{1}\left|\frac{x s}{2}\right|^{2} \mathrm{~d} s\right) \mathrm{d} x=\frac{1}{36}$.

For every $u \in A$ and $v \in B$, there holds the inequality

$$
\frac{1}{5}=\frac{\int_{0}^{1} K(x, s) 2 s \mathrm{~d} s}{2 g(x)} \leq \inf \left\{\frac{\int_{0}^{1} K(x, s)(u(s)-v(s)) \mathrm{d} s}{2 g(x)}, x \in(0,1]\right\}
$$

Let us put $t_{0}=1 / 5$ and from the inequality (15) for $p=2$, we obtain that the inequality

$$
\begin{aligned}
J_{2} & =\left(1+\frac{\int_{0}^{1} K(x, s)(u(s)-v(s)) \mathrm{d} s}{2 g(x)}\right)^{2} \\
& \leq\left(1+t_{0}\right)\left(1+\frac{1}{t_{0}}\left(\frac{\int_{0}^{1} K(x, s)(u(s)-v(s)) \mathrm{d} s}{2 g(x)}\right)^{2}\right)
\end{aligned}
$$


holds true for every $x \in(0,1]$. Consequently, we get the chain of inequalities

$$
\begin{aligned}
J_{3} & =\tilde{M}(T u-T v) \\
& =\int_{0}^{1}\left(2 g(x)+\int_{0}^{1} K(x, s)(u(s)-v(s)) \mathrm{d} s\right)^{2} \mathrm{~d} x \\
& \leq\left(1+t_{0}\right) \int_{0}^{1}(2 g(x))^{2} \mathrm{~d} x+\int_{0}^{1}\left(\frac{1+t_{0}}{t_{0}}\right)\left(\int_{0}^{1} K(x, s)(u(s)-v(s)) \mathrm{d} s\right)^{2} \mathrm{~d} x \\
& \leq\left(1+t_{0}\right) a+\frac{1+t_{0}}{t_{0}} \int_{0}^{1}\left(\int_{0}^{1}|K(x, s)|^{2} \mathrm{~d} s\right)\left(\int_{0}^{1} \mid\left(u(s)-\left.v(s)\right|^{2} \mathrm{~d} s \mid\right) \mathrm{d} x\right. \\
& \leq\left(1+t_{0}\right) a+\frac{1+t_{0}}{t_{0}}\left(\int_{0}^{1} \int_{0}^{1}|K(x, s)|^{2} \mathrm{~d} s \mathrm{~d} x\right) \widetilde{M}(u-v) \\
& =\left(1+t_{0}\right) a+\frac{1+t_{0}}{t_{0}} c \widetilde{M}(u-v) \\
& =\frac{1+t_{0}}{t_{0}} c \tilde{M}(u-v)+\left(1+t_{0}\right) \frac{a}{b} d_{\widetilde{M}} .
\end{aligned}
$$

For $t_{0}=1 / 5$, there hold $\frac{1+t_{0}}{t_{0}} c \in(0,1)$ and the equality $\left(1+t_{0}\right) \frac{a}{b}+\frac{1+t_{0}}{t_{0}} c=1$. Consequently, we get that any $u \in A, v \in B$ there holds the inequality

$$
\widetilde{M}(T u-T v) \leq \frac{1+t_{0}}{t_{0}} c \widetilde{M}(u-v)+\left(1-\frac{1+t_{0}}{t_{0}} c\right) d_{\widetilde{M}} .
$$

(c4) Let $u \in A$. Then from the inequalities $x \leq u(x) \leq n x$, we obtain $-n x \leq-g(x)-n \int_{0}^{x} K(x, s) s \mathrm{~d} s \leq$ $T u(x) \leq-g(x)-\int_{0}^{1} K(x, s) s \mathrm{~d} s=-x$. Therefore, $-n x \leq T u(x) \leq-x$, i.e., $T(A) \subseteq B$.

The inclusion $T(B) \subseteq A$ is obtained in a similar fashion.

Thus from Theorem 6.4 , it follows that there exists a unique $\widetilde{M}$-best proximity point $u \in A$, such that for any $u_{0} \in A$, the sequence $\left\{T^{2 n} u_{0}\right\}_{n=1}^{\infty}$ is $\widetilde{M}$-convergent to $u$. It is easy to observe that the function $x$ is a $\widetilde{M}$-best proximity point of $T$ in $A, T(x)=-x, T^{2}(x)=T(-x)=x$.

\section{Concluding remarks}

We would like to pose some open questions. Is it possible to replace the condition for uniform continuity of the function modular $\rho$ in Theorem 3.2 with just a continuity? Is it possible to replace (UC1) with (UC2) in Theorem 3.2?

We expect that all generalizations for best proximity points for different kinds of maps will be possible to be done for modular spaces.

Acknowledgments I would like to thank the anonymous reviewers for their careful reading of my manuscript and for their suggestions that improved the text and helped to correct some gaps in the proofs.

Open Access This article is distributed under the terms of the Creative Commons Attribution 4.0 International License (http:// creativecommons.org/licenses/by/4.0/), which permits unrestricted use, distribution, and reproduction in any medium, provided you give appropriate credit to the original author(s) and the source, provide a link to the Creative Commons license, and indicate if changes were made.

\section{References}

1. Chen, S.: Geometry of Orlicz spaces. Dissertat. Math. 356, 4205 (1996)

2. Eldred, A.; Veeramani, P.: Existence and convergence of best proximity points. J. Math. Anal. Appl. 323(2), 1001-1006 (2006)

3. Kaminska, A.: On uniform convexity of Orlicz spaces. Indag. Math. 44(1), 27-36 (1982)

4. Khamsi, M.A.; Kozlowski, W.M.: Fixed Point Theory in Modular Function Spaces. Springer, Berlin (2015)

5. Khamsi, M.A.; Kozlowski, W.M.; Reich, S.: Fixed point theory in modular function spaces. Nonlinear Anal. 14(11), 935-953 (1990) 
6. Khamsi, M.A.; Kozlowski, W.M.; Shutao, C.: Some geometrical properties and fixed point theorems in Orlicz spaces. J. Math. Anal. Appl. 155, 393-412 (1991)

7. Kirk, W.; Srinivasan, P.; Veeramani, P.: Fixed points for mappings satisfying cyclical contractive conditions. Fixed Point Theory 4, 79-189 (2003)

8. Kozlowski, W.M.: Notes on modular function spaces I. Comment. Math. 28, 91-104 (1988)

9. Kozlowski, W.M.: Notes on modular function spaces II. Comment. Math. 28, 105-120 (1988)

10. Kozlowski, W.M.: Modular Function Spaces. Marcel Dekker Inc, New York (1988)

11. Kozlowski, W.M.: Advancements in fixed point theory in modular function spaces. Arab. J. Math. 1, 477-494 (2012)

12. Lindenstrauss, J.; Tzafriri, L.: Classical banach spaces I. Sequence Spaces. Springer, Berlin (1977)

13. Lindenstrauss, J.; Tzafriri, L.: Classical banach spaces II. Function Spaces. Springer, Berlin (1979)

14. Musielak, J.; Orlicz, W.: On modular spaces. Stud. Math. 18, 591-597 (1959)

15. Nakano, H.: Modular Semi-Ordered Spaces. Maruzen, Tokyo (1950)

16. Rao, M.; Ren, Z.: Theory of Orlicz Spaces. Marcel Dekker Inc, New York (1991)

17. Rao, M.; Ren, Z.: Applications of Orlicz Spaces. Marcel Dekker Inc, New York (2002) 